\title{
Innovation drug approvals based on a bridging study: from concept to practice
}

\author{
Tao Wang ${ }^{1}$, Xiao $\mathrm{Cao}^{2}$, Yingjian $\mathrm{He}^{3}$, Xiaoyuan $\mathrm{Chen}^{4}$ \\ ${ }^{1}$ Department of Oncology, the Fifth Medical Center of Chinese PLA General Hospital, Beijing, China; ${ }^{2}$ Medical Affairs, Zai Lab (Shanghai) Co., \\ Ltd, Shanghai, China; ${ }^{3}$ Key Laboratory of Carcinogenesis and Translational Research (Ministry of Education/Beijing), Breast Center, Peking \\ University Cancer Hospital \& Institute, Beijing, China; ${ }^{4}$ Tsinghua Clinical Research Institute (TCRI), School of Medicine, Tsinghua University, \\ Beijing, China \\ Contributions: (I) Conception and design: T Wang, X Chen; (II) Administrative support: X Cao; (III) Provision of study materials or patients: \\ T Wang; (IV) Collection and assembly of data: T Wang, X Chen; (V) Data analysis and interpretation: T Wang, Y He; (VI) Manuscript writing: All \\ authors; (VII) Final approval of manuscript: All authors. \\ Correspondence to: Xiaoyuan Chen. Tsinghua Clinical Research Institute (TCRI), School of Medicine, Tsinghua University, Beijing, China. \\ Email: cxya02648@mail.tsinghua.edu.cn.
}

\begin{abstract}
Many factors (pathological subtype differences, etiology, clinical practice, etc.) may cause ethnic sensitivities in drugs. For drugs approved in one region, their differences among ethnic groups and the potential impacts of such differences on their safety and efficacy must be explained before seeking approvals in another region. Despite potential ethnic sensitivities, if pharmaceutical companies are required to repeat the clinical research and development process in various countries, it will result in waste of resources and delays in drug approval. To address this issue, the International Conference on Harmonization (ICH) published a guideline entitled Ethnic Factors in the Acceptability of Foreign Clinical Data, known as ICH E5, in 1998. With an attempt to offer guidance on the monitoring and research \& development (R\&D) of innovation drugs, the concept of bridging studies was for the first proposed, which allows the adequate assessment of ethnic differences in safety, efficacy, dosage, or dose regimen of an innovation drug, and meanwhile minimizes the duplication of clinical data in two different regions to speed up the licensing of the drug in the new region. Here we take breast cancer as an example to describe the concept, types, strategies, statistical methods, and clinical practice of bridging studies, focusing on their development in China.
\end{abstract}

Keywords: Bridging study; ethnic sensitivity; bridging strategy (BG); breast cancer

Received: 29 December 2021; Accepted: 13 January 2022.

doi: $10.21037 /$ tbcr-21-43

View this article at: https://dx.doi.org/10.21037/tbcr-21-43

\section{How we define and understand bridging studies \\ Concept of bridging studies}

A bridging study is defined as an additional study performed in a new region to provide pharmacokinetics (PK), pharmacodynamics (PD), or clinical data on efficacy, safety, dosage, and dose regimen of an innovation drug in the new region, which will allow extrapolation of foreign clinical data to the population in the new region (1).

\section{Ethnic sensitivity}

Ethnic factors may influence the efficacy and safety of drugs. In the International Conference on Harmonization (ICH) E5 guidelines, ethnic differences are classified as intrinsic (based on genetics or physiology) and extrinsic (based on cultural or environmental characteristics). Drugs are classified as ethnically sensitive or insensitive, according to PK differences between races and ethnic groups. And, 
the sensitivity could be evaluated by a number of factors, such as PK and PD (2). ICH E5 lists the properties of drugs susceptible and not susceptible to ethnic factors. These key properties include linear $\mathrm{PK}, \mathrm{PD}$, therapeutic range, protein binding potential, interactions, genetic polymorphism, inter-subject variability, a systemic mode of action, and potential for inappropriate use. Although ethnic differences may cause differences in safety, efficacy, dosage, or dose regimen in a new region, many drugs have comparable $\mathrm{PK}$ and PD characteristics and clinical effectiveness across regions. A study in Taiwan Province of China found that complete clinical data containing Asian PK data and clinical efficacy data were present in many successful bridging studies; under some conditions, it is satisfactory that ethnic concerns for safety and efficacy might be answered by a phase 4 study (3).

\section{Definitions of bridging data package}

The ICH E5 guideline suggests the regulatory authority of the new region assesses the ability to extrapolate foreign data based on the bridging data package, which comprises: (I) selected information from the Complete Clinical Data Package (CCDP) that applies to the population of the new region, including $\mathrm{PK}$ data and any preliminary $\mathrm{PD}$ and dose-response data; and (II) if needed, a bridging study to extrapolate the foreign efficacy data and/or safety data to the new region.

\section{Types of bridging studies}

The types of bridging study depend on the ethnic differences between the new and original regions. Ethnic sensitivity determines the need to conduct local clinical trials. A bridging study may be a phase I PK/PD study or a phase II/III randomized controlled clinical trial, if the drug is racially sensitive and geographically dissimilar. At the same time, if there is enough clinical trial experience and drug classification is familiar, phase I PD study is required. If the drug is racially sensitive, but the medical practice is different in the two places, and the classification of the drug is not familiar in the two places, randomized controlled trial (RCT) tests should be performed (4).

The following situations do not require a bridge test: drugs are not sensitive to racial factors, and the requirements for drug use and clinical trials are broadly similar in both places; drugs are sensitive to racial factors, but they are racially similar in both places, and there is sufficient pharmacological and clinical experience to indicate that the efficacy, safety, dose and administration schedule of the drugs are similar in both places. The specific type should be decided if required.

\section{Bridging strategy (BG)}

A bridging study is often required to assess the suitability of extrapolating foreign data during the registration of a drug. The implementation and application processes of such a study are called BGs (5). There are four BG types: (I) standalone PK studies and dose-response clinical trials in healthy subjects; (II) stand-alone PK studies and phase II doseresponse clinical trials in both healthy subjects and patients; (III) without stand-alone PK study, but with PK studies included in clinical trials; and (IV) with both stand-alone PK studies and PK studies included in clinical trials (6).

BG in Japan has made remarkable progress. A study investigated antitumor drugs approved in Japan from April 2001 to July 2014, and concluded that "Japan's participation in global clinical trials" and "bridging strategies" were potential factors that reduce drug lag (7). Also, Kogure et al. investigated the potential factors that influenced submission lag (SL) and compared the differences in SL among early-initiation BG strategy, late-initiation BG strategy, and global trial (GT) strategy. They found SL in the GT strategy, and that in the early-initiation BG strategy was significantly shorter than that in the late-initiation BG strategy. Thus, they urged the early utilization of BG to avoid future drug lag (8).

\section{Statistical methods in bridging studies}

The evaluation of bridging justification does not use clinical data from abroad to verify the effectiveness of overall treatment, but to explain that small-scale trials conducted in the new region are similar to studies conducted in the original region, thus achieving the purpose of extrapolating clinical data from the original region to the new region. "Similarity" is a crucial problem in bridge test, but the methodology system is not perfect. First, researches on "similarity" can be divided into classical statistical method (frequency method) and Bayesian statistical method from methodology. Second, from the different understanding of similarity, it can be divided into necessity evaluation, similarity evaluation, equivalence/non-inferiority evaluation; Third, the idea of using multi-center clinical trials includes sequential design, weighted compromise scheme, multicenter hierarchical model, etc. (9). 
Using pharmacological endpoints as study indicators, the similarity of different ethnic data is evaluated, usually using graphical and modeling methods. Shih [2001] interpreted the similarity as consistency between research centers. Under this definition, in 2001 Shih proposed a sequential procedure to test consistency between foreign studies and bridging studies (10). Recently, the pi-based conformance test proposed by Pan et al. provided a novel and practical statistical method to evaluate the conformance of the results of biosimilar bridging trials with the results of biosimilar trials worldwide. This test could be used to establish the necessary Bridges between global biosimilar research data and new regions or countries (11).

Liu et al. and Wang et al. proposed similarities based on equivalence and non-inferiority in the literature $(12,13)$. Liu et al. proposed a method to evaluate the average bioequivalence between generic drugs in the new region and innovative drugs in the original region using generic drugs in the original region as bridging reference agents (14).

\section{Reproducibility/generalizability}

Reproducibility/generalizability can be calculated, and ethnic sensitivity is measured to determine the types of bridging studies. Shao and Chow [2002] proposed a sensitivity index to assess the reproducibility probability, used to measure ethnic sensitivity and categorize bridging studies (15). The reproducibility probability is the probability of repeating the results of the original trial in a new region. When there is no clear evidence that ethnic differences between two regions impact drug efficacy, the calculated reproducibility probability can be used to determine whether a bridging study is warranted. Generalizability probability refers to the probability of efficacy in the new region when existing information indicates that there may be differences in efficacy between the new region and the original region.

\section{Weighted Z test}

Lan et al. proposed weighted Z-tests in medical research in 2005. The weighted $Z$-tests can be applied to the sample size re-estimation or analysis of bridging studies based on the weights selected (16). In 2012, Huang et al. proposed a new method for the design and sample size consideration for a simultaneous global drug development program (SGDDP) using weighted z-tests (17). In 2012, Tsou et al. proposed another weighted evidence approach of bridging studies, drawing on Lan's weighted Z-tests. The authors considered the overall treatment effect by combining the weighted effects attained in the original and bridging regions, rather than the weighted combinations of two test statistics (18). In practice, attention should be paid to the expansion of type I error, and the size of type I error should meet the regulatory requirements of drug approval in relevant regions.

\section{Group sequential designs}

Hsiao et al. considered bridging studies as clinical trials conducted in two phases (in the original and new regions) under a unified framework. A bridging study, which may be conducted simultaneously with drug research \& development (R\&D), can be considered a subgroup study in the trial. Many patients are recruited as the subgroup in the new region, and the bridging study should be implemented according to the research protocol applied in the original region $(19,20)$.

\section{Bayesian approach}

Liu et al., in 2002, introduced the classical Bayesian method into the analysis of bridge tests, using the posterior probability of population parameters to construct the corresponding similarity (21). The method was improved in 2004 (22) to better assess the similarity between the new and original regions. Hsiao et al. improved the definition of prior information based on the classical Bayesian method and proposed the mixed prior Bayesian method (23). The main difficulties in the application of Bayesian method in bridging study are the determination of prior information or the utilization degree of existing information in the original region, and the estimation of type I errors in decision making by posterior probability. Recently, Zeng et al. have provided a new method for the design and analysis of bridging studies that controls for the average type I error of bridging studies over all possibilities of evidence from foreign studies (24).

According to the above introduction, we found that there are various statistical methods for bridge test, but all of them have difficulties in operation, and there is no unified and operable international standard. Standards are closely related with national policies and regulations.

\section{Registration requirements for bridging studies in China}

Although the concept of "bridge test" has not been 
explicitly put forward in The Drug Registration Management Measures issued in 2002, similar management requirements have been set up. According to the drug registration regulation (2007 version), foreign applicants to conduct international multi-center drug clinical trials in China, they shall apply to the State Food and Drug Administration in accordance with these Measures, and the drugs used for clinical trials shall be in drugs registered overseas or drugs that have entered phase II or phase III clinical trials. Thus, conducting bridging studies was a reasonable choice. In 2015, China issued guidelines for International Multi-center Drug Clinical Trials, which improved the approval of drug clinical trials, and allow concurrent clinical trials of innovation drugs that are not yet on the market in China after approval. In 2017, the China Food and Drug Administration (CFDA) released the Decision of the State Food and Drug Administration on Adjusting Matters Relating to the Registration of Imported Drugs (State Food and Drug Administration Order No. 35). This allows the implementation of international multicenter clinical trials in China, permits simultaneous phase I clinical trials to be carried out in China, and deletes the requirement that "drugs for clinical trials must have been registered overseas or entered phase II or phase III clinical trials". After completing the international multicenter clinical trials of a drug in China, the applicant can directly apply for the licensing of the drug. The above new requirements for registration were further clarified in the Technical Guidelines for the Acceptance of Overseas Clinical Trial Data for Drugs [2018], the Clinical Technical Requirements for Drugs Marketed Overseas but not in Mainland China [2020], and the 2020 version of Measures for the drug registration regulation. The past decade has seen the transition of the development and registration strategies for imported drugs in China from "conducting bridging studies after drug approval overseas" to "China joining multiregional clinical trials before global approval "; in the absence of potential racial/ethnic disparities, clinical trials in China may be exempted (usually for products already marketed overseas and urgently needed in clinical settings). China now directly joins phase III (or even earlier) international clinical trials, or pre-set a window for the number of Chinese patients enrolled in a global phase III trial to meet China's registration requirements. Notably, the diversification of new drug R\&D in China and the emergence of multiple innovative models, including License-in/out, have made the new drug registration strategies more complex, and bridging studies will play new roles. The globalization of drug $R \& D$ brings not only the convenience of innovation drug application, but also the diversification of regulatory systems in different countries and regions. Up to now, there is still a lack of internationally recognized definitions and standard requirements for issues such as efficacy, safety, and ethnic sensitivities.

At the same time, with the rise of domestic drug research and development, the trend of new drug license out has become more and more obvious. When local products enter the markets of other countries, it is necessary for relevant companies and departments to conduct international dialogues on scientific and regulatory issues.

\section{Development and application of bridging studies in China}

\section{Bridging studies for drugs in China}

Many countries have adopted bridging studies in the R\&D of innovation drugs. The BG shortens development cycles, lowers R\&D costs, and promotes globalization, enabling rapid approval of new drugs in new regions. Supported by these changes, 43 imported innovation cancer drugs were approved in China between 2010 and 2020. From 2010 to 2020, 13 innovation drugs approved by the Food and Drug Administration (FDA) after 2006 have undergone bridging trials and have been approved in China; 27 innovation drugs have been approved in China through multi-regional clinical trial (MRCT). Compared with the bridging test, MRCT significantly shortens the drug lag, but at the beginning of this century, bridging trials also made a significant contribution to the approval of innovation drugs (25).

\section{Application of bridging studies for breast cancer}

Breast cancer is the most common malignant tumor among women in China, with high malignancy, rapid disease progression, prone to lymph node metastasis, and poor prognosis. With the advancement of molecular biology, more molecularly targeted drugs with higher precision have been applied in the clinical treatment of breast cancer.

The rapid approval of targeted drugs for breast cancer in China also depends highly on bridging studies. As early as 2002, Professor Sun Yan conducted a clinical study on the treatment of $A B C$ with trastuzumab, and had already demonstrated the bridging concept. According to the response rate $(21 \%)$ of trastuzumab alone in the treatment of $\mathrm{ABC}$ published at the annual meeting of the 
American Society of Clinical Oncology (ASCO) in 1998 and other recent reports on trastuzumab, Professor Sun Yan conducted a multi-center verification clinical study in China. The results showed that of the 31 patients, after trastuzumab treatment, 2 cases were complete response (CR), 6 cases were partial response (PR), 7 cases were stable disease (SD), and progression disease (PD) in 16 cases, with response rate of $25.8 \%$. Although this study was not using typical bridging test, it was a precedent in the bridging research of breast cancer drugs in China (26).

The strategic development of innovation drug $R \& D$ pattern has evolved from post-marketing bridging trials to MRCT. The 1998 ICHE5 guidelines, which assess racial differences and sensitivities of drugs, provide guidance for continuous bridging tests. To E5 QA, it was proposed that MRCT could answer the question of racial differences. The E17 guidelines further confirmed the importance of MRCT, which proposed to look at global data first and then evaluate regional consistency.

Selective cyclin-dependent kinase 4 and 6 (CDK4/6) inhibitors are highly effective targeted agents for hormone receptor (HR)-positive, HER2-negative luminal breast cancer. MONARCH-2 was a global, randomized, placebocontrolled, double-blind phase III trial of abemaciclib plus fulvestrant $v s$. placebo plus fulvestrant for treating women with HR-positive, HER2-negative ABC that progressed during endocrine therapy (ET). Results showed that abemaciclib plus fulvestrant significantly prolonged PFS (16.4 vs. 9.3 months, HR: 0.553, $\mathrm{P}=0.0000001)$ and OS (46.7 vs. 37.3 months, HR: 0.757, $\mathrm{P}=0.0137$ ) (27). The US FDA has approved abemaciclib combined with fulvestrant to treat women with HR-positive, HER2negative, locally advanced, or metastatic breast cancer with disease progression following ET. MONARCH-3 is a randomized, phase III, double-blind study of abemaciclib/ placebo plus nonsteroidal aromatase inhibitor (AI) to treat postmenopausal women with HR+, HER2- locally ABC with no prior systemic therapy (28). The abemaciclib arm had a significantly longer median PFS than the placebo arm (28.18 vs. 14.76 months). Therefore, the US FDA approved Abemaciclib combined with AI as initial ET for patients with HR-positive, HER2-negative locally advanced, or metastatic breast cancer in postmenopausal women.

To accelerate the approval of abemaciclib in China, The MONARCH plus study was conducted combining the design of MONARCH-2 and MONARCH-3 (29). It was an international multicenter phase III clinical trial led by Chinese researchers. A total of 463 patients were enrolled, among whom $80 \%$ were Chinese patients. The subjects were divided into two cohorts: cohort A and cohort B (abemaciclib + fulvestrant $v s$. placebo + fulvestrant). According to the study design, the primary purpose of cohort $\mathrm{B}$ was to provide a conformance comparison with MONARCH 2. In cohort $\mathrm{B}$, the median PFS was 11.47 months in the abemaciclib + fulvestrant arm vs. 5.59 months in the placebo + fulvestrant arm, with objective response rate (ORR) rates being 50.0\% and $10.5 \%$ respectively. These results demonstrated the definitive efficacy of abemaciclib in combination with ET for HR-positive ABC in China, which was consistent with the benefit trends in global studies. In December 2020, the National Medical Products Administration (NMPA) of China approved abemaciclib for the treatment of HRpositive, HER2-negative locally advanced/metastatic breast cancer, either in combination with an aromatase inhibitor as the initial ET for postmenopausal women, or in combination with fulvestrant for patients who have experienced disease progression after prior ET. From the official start of the MONARCH Plus study in 2016 to the approval of abemaciclib in China, only a short period of 4 years has elapsed in between. The BG has greatly shortened the drug $\mathrm{R} \& \mathrm{D}$ process, making it possible for efficacious and safe drugs to be marketed in China promptly.

Here is another typical case of the application of BG in the field of breast cancer. Margetuximab is an Fc-engineered, immune-activated anti-HER2 immunoglobulin G1 (IgG1) monoclonal antibody with the same epitope specificity as trastuzumab. It modified the affinity with $\mathrm{Fc}$ receptors on the surface of immunocytes, increasing both intrinsic immunity (ADCC effect) and adaptive immunity. Rugo et al. conducted a global, multicenter phase III, randomized, open-label SOPHIA trial to compare the efficacy of margetuximab with that of trastuzumab (30). Totally 536 patients were enrolled at 166 sites in 17 countries from August 26, 2015, to October 10, 2018. They were randomized to receive margetuximab + chemotherapy (margetuximab group) or trastuzumab + chemotherapy (trastuzumab group). Margetuximab improved primary endpoint PFS over trastuzumab with $24 \%$ relative risk reduction. After the second planned interim analysis of 270 deaths, the median overall survival (OS) was 21.6 months with margetuximab vs. 19.8 months with trastuzumab. The safety profile was comparable between these two groups.

Investigators have attempted to conduct bridging studies to bring the benefit of margetuximab to Chinese HER2+ patients early. In February and May 2020, respectively, two studies evaluating margetuximab combined with 
chemotherapy for Chinese patients with HER2+ metastatic breast cancer were conducted in China. CTR20200242 is a phase I clinical trial of 16-20 patients to evaluate the PK profile of margetuximab in combination with chemotherapy in Chinese HER2-positive patients with metastatic breast cancer that did not respond to prior anti-HER2 targeted therapy. The primary endpoints were PK parameters after intravenous administration of margetuximab, and the secondary endpoints included drug efficacy and safety. CTR2020024 is a phase II, open-label, randomized, controlled trial. The study was designed to enroll 120 patients with histologically confirmed HER2-positive metastatic breast cancer who had received prior secondor later-line anti-HER2 targeted therapy at the metastatic disease stage. The trial's objective was to evaluate the efficacy and safety of margetuximab in combination with chemotherapy versus trastuzumab plus chemotherapy in Chinese patients with advanced HER2-positive metastatic breast cancer whose diseases had progressed following prior anti-HER2 therapy. The primary endpoint was PFS, and the secondary endpoints included OS, ORR, duration of response (DoR), and clinical benefit rate (CBR). Notably, considering the differences in clinical practice between Chinese and Western countries, the CTR2020024 study did not require prior treatment with pertuzumab and allowed prior treatment with pyrotinib. Thus, it can be expected that the patients enrolled in this study will be closer to the real-world settings in China. We expect the findings of these two studies will accelerate margetuximab launch to benefit Chinese breast cancer population.

\section{Summary and prospects}

The BG shows advantages in extrapolating foreign clinical trial data, identifying racial differences, reducing duplicate trials, and shortening the review time for the innovation drug. Bridging studies are additional studies performed in a new region to provide $\mathrm{PK}, \mathrm{PD}$, or clinical data on a new drug's safety, efficacy, dosage, or dose regimen in the new region, which will allow extrapolation of the foreign clinical data the population in the new region. Bridging studies have been successfully conducted in some countries and regions, and have become important for new drug applications.

As the R\&D of new drugs in China has been diversified, bridging studies in China now have multiple models and new definitions. The feasible strategies may include optimized pharmaceutical regulations and technologies, early initiation of BGs, proactive participation in global
$\mathrm{R} \& \mathrm{D}$ trials, and even pre-defined windows in GTs to meet Chinese registration requirements. These strategies will be of great significance to promote the development of China's pharmaceutical industry and bring great survival benefits to cancer patients.

However, the BG faces some disadvantages and challenges. Although the clinical needs of bridging trials in China are imminent, their development still requires scientific management and supervision. What kind of BG and bridging results are accepted, in addition to the scientific considerations of ethnic sensitivities analysis, clinical needs and clinical advantages are important considerations, as well as a trade-off between science and regulation. On the one hand, we hope to have a more flexible, efficient and interactive regulatory system so that decisions can be made when data is insufficient. But on the other hand, the hope is that drug research will be rigorous and independent. Therefore, in the future, how to explore some good methods, or help clinical workers to make some new tools and methods, to help the regulatory authorities to formulate standards, this may be the direction of every medical worker's future efforts. With the rapid development and application of innovation drugs today, there are still many challenges in global $\mathrm{R} \& \mathrm{D}$ bridging research design and implementation including sample size distribution in different countries, etiology, pathological subtypes, how to analyze inconsistent data in clinical practice, and how to coordinate when conducting multi-country research are all problems that researchers are committed to solving.

Even so, the value and significance of the bridge test is indisputable. With the globalization of drugs, various bridging trials will help patients get in touch with more types of innovation drugs faster, and provide the greatest help for the cure of the disease.

\section{Acknowledgments}

We want to thank the Editor and anonymous referees for their constructive suggestions.

Funding: None.

\section{Footnote}

Peer Review File: Available at https://tbcr.amegroups.com/ article/view/10.21037/tbcr-21-43/prf

Conflicts of Interest: All authors have completed the ICMJE uniform disclosure form (available at https://tbcr. 
amegroups.com/article/view/10.21037/tbcr-21-43/coif). The authors have no conflicts of interest to declare.

Ethical Statement: The authors are accountable for all aspects of the work in ensuring that questions related to the accuracy or integrity of any part of the work are appropriately investigated and resolved.

Open Access Statement: This is an Open Access article distributed in accordance with the Creative Commons Attribution-NonCommercial-NoDerivs 4.0 International License (CC BY-NC-ND 4.0), which permits the noncommercial replication and distribution of the article with the strict proviso that no changes or edits are made and the original work is properly cited (including links to both the formal publication through the relevant DOI and the license). See: https://creativecommons.org/licenses/by-nc-nd/4.0/.

\section{References}

1. ICH, International Conference on Harmonization Tripatite Guidence E5. Ethnic factors in the acceptability of foreign clinical data. The U.S. Fed Reg 1998;83:31790-6.

2. Gupta SK. Implications of ICH-E5: Assessment of drug's sensitivity to ethnic factors and necessity of a bridging study for global drug development. Perspect Clin Res 2011;2:121-3.

3. Hsiao CL, Lin YL, Hsu LF, et al. Ten-year experience of the evaluation of ethnic sensitivity data. Drug Information Journal 2011;45:717-24.

4. Liu JP, Chow SC. Bridging studies in clinical development. J Biopharm Stat 2002;12:359-67.

5. Uyama Y, Shibata T, Nagai N, et al. Successful bridging strategy based on ICH E5 guideline for drugs approved in Japan. Clin Pharmacol Ther 2005;78:102-13.

6. Tanaka M, Nagata T. Characteriation of clinical data packages using foreign data in new drug applications in Japan. Clin Pharmacol Ther 2008;84:340-6.

7. Maeda H, Kurokawa T. Recent trends for drug lag in clinical development of oncology drugs in Japan: does the oncology drug lag still exist in Japan? Int J Clin Oncol 2015;20:1072-80

8. Kogure S, Koyama N, Hidaka S. Utilization of the Bridging Strategy for the Development of New Drugs in Oncology to Avoid Drug Lag. J Clin Pharmacol 2017;57:1479-90.

9. Chow SC, Chiang C, Liu JP, et al. Statistical methods for bridging studies. J Biopharm Stat 2012;22:903-15.

10. Shih WJ. Clinical trials for drug registrations in AsianPacific countries: proposal for a new paradigm from a statistical perspective. Control Clin Trials 2001;22:357-66.

11. Pan Z, Huang W, Pan J, et al. Design and Analysis of a Biosimilar Bridging Study with a Prediction Interval-Based Consistency Test. Ther Innov Regul Sci 2021;55:765-72.

12. Liu JP, Hsueh H, Chen JJ. Sample size requirements for evaluation of bridging evidence. Biometrical Journal 2002;44:969-81.

13. Wang SJ, Hung HM. TACT method for non-inferiority testing in active controlled trials. Stat Med 2003;22:227-38.

14. Liu JP. Bridging bioequivalence studies. J Biopharm Stat 2004;14:857-67.

15. Shao J, Chow SC. Reproducibility probability in clinical trials. Stat Med 2002;21:1727-42.

16. Lan KK, Soo Y, Siu C, et al. The use of weighted Z-tests in medical research. J Biopharm Stat 2005;15:625-39.

17. Huang Q, Chen G, Yuan Z, et al. Design and sample size considerations for simultaneous global drug development program. J Biopharm Stat 2012;22:1060-73.

18. Tsou HH, Tsong Y, Liu JT, et al. Weighted evidence approach of bridging study. J Biopharm Stat 2012;22:952-65.

19. Hsiao CF, Xu JZ, Liu JP. A group sequential approach to evaluation of bridging studies. J Biopharm Stat 2003;13:793-801.

20. Hsiao CF, Xu JZ, Liu JP. A two-stage design for bridging studies. J Biopharm Stat 2005; 15:75-83.

21. Liu JP, Hsiao CF, Hsueh H. Bayesian approach to evaluation of bridging studies. J Biopharm Stat 2002;12:401-8.

22. Liu JP, Hsueh H, Hsiao CF. A Bayesian noninferiority approach to evaluation of bridging studies. J Biopharm Stat 2004; 14:291-300.

23. Hsiao CF, Hsu YY, Tsou HH, et al. Use of prior information for Bayesian evaluation of bridging studies. J Biopharm Stat 2007;17:109-21.

24. Zeng D, Pan Z, Lin DY. Design and analysis of bridging studies with prior probabilities on the null and alternative hypotheses. Biometrics 2020;76:224-34.

25. Li G, Liu Y, Xie C, et al. Characteristics of expedited programmes for cancer drug approval in China. Nat Rev Drug Discov 2021;20:416.

26. Sun Y, Li L, Song S, et al. Clinical verification results of trastuzumab for injection in the treatment of advanced breast cancer. Chinese Journal of Oncology 2003;25:581-3.

27. Sledge GW Jr, Toi M, Neven P, et al. The Effect of 
Abemaciclib Plus Fulvestrant on Overall Survival in Hormone Receptor-Positive, ERBB2-Negative Breast Cancer That Progressed on Endocrine TherapyMONARCH 2: A Randomized Clinical Trial. JAMA Oncol 2020;6:116-24.

28. Johnston S, Martin M, Di Leo A, et al. MONARCH 3 final PFS: a randomized study of abemaciclib as initial therapy for advanced breast cancer. NPJ Breast Cancer 2019;5:5.

doi: $10.21037 /$ tbcr-21-43

Cite this article as: Wang T, Cao X, He Y, Chen X. Innovation drug approvals based on a bridging study: from concept to practice. Transl Breast Cancer Res 2022;3:2.
29. Zhang QY, Sun T, Yin YM, et al. MONARCH plus: abemaciclib plus endocrine therapy in women with HR+/HER2- advanced breast cancer: the multinational randomized phase III study. Ther Adv Med Oncol 2020;12:1758835920963925.

30. Rugo HS, Im SA, Cardoso F, et al. Efficacy of Margetuximab vs Trastuzumab in Patients With Pretreated ERBB2-Positive Advanced Breast Cancer: A Phase 3 Randomized Clinical Trial. JAMA Oncol 2021;7:573-84. 